\title{
Nutrient cross-feeding in the microbial world
}

\section{Erica C. Seth and Michiko E. Taga*}

Department of Plant and Microbial Biology, University of California, Berkeley, Berkeley, CA, USA

\section{Edited by:}

Luis Raul Comolli, Lawrence Berkeley National Laboratory, USA

\section{Reviewed by:}

Yasuyoshi Sakai, Graduate School of Agriculture, Kyoto University, Japan Ulas Karaoz, Lawrence Berkeley

National Laboratory, USA

\section{*Correspondence:}

Michiko E. Taga, Department of Plant and Microbial Biology, University of California, Berkeley, 111 Koshland Hall, Berkeley, CA 94720-3102, USA

e-mail: taga@berkeley.edu
The stability and function of a microbial community depends on nutritional interactions among community members such as the cross-feeding of essential small molecules synthesized by a subset of the population. In this review, we describe examples of microbemicrobe and microbe-host cofactor cross-feeding, a type of interaction that influences the forms of metabolism carried out within a community. Cofactor cross-feeding can contribute to both the health and nutrition of a host organism, the virulence and persistence of pathogens, and the composition and function of environmental communities. By examining the impact of shared cofactors on microbes from pure culture to natural communities, we stand to gain a better understanding of the interactions that link microbes together, which may ultimately be a key to developing strategies for manipulating microbial communities with human health, agricultural, and environmental implications.

Keywords: nutrient cross-feeding, cofactor, microbial interactions, corrinoid, microbial communities

\section{INTRODUCTION}

Life in the microbial world exists as a dynamic network of interactions among microbes that fuels a complex web of interconnected metabolisms (Faust and Raes, 2012). Ignorance of what microbes gain via these interactions impedes our ability to cultivate the vast majority of microbes (Leadbetter, 2003; Tyson and Banfield, 2005). In addition, by failing to elicit a microbe's full range of metabolic responses to the presence of other organisms, the metabolic potential of microbes grown in isolation may not accurately reflect a microbe's ecological role (Moller et al., 1998; Traxler et al., 2013). Three broad categories of nutritional interactions that govern a microbe's ability to carry out specific forms of metabolism within a microbial community are illustrated in Figure $\mathbf{1 .}$

The ability to compete for nutrients can determine whether a microbe will be able to persist in a particular niche (Hibbing et al., 2010; Johnson et al., 2012; Figure 1A). Specialized ability to gain access to a limiting nutrient (for example, iron acquisition via siderophore production) may give a microbe a competitive advantage in colonization. In contrast, metabolic cooperation between microbes engaged in syntrophic partnerships can allow access to substrates that neither microbe could metabolize alone (Figure 1B). For example, the presence of a partner that actively consumes intermediates such as hydrogen $\left(\mathrm{H}_{2}\right)$ allows secondary fermentation of products such as propionate to become energetically favorable (Sieber et al., 2012; Morris et al., 2013). Finally, nutrient cross-feeding - the production of a molecule such as a vitamin or amino acid that is used by both the producing organism and other microbes in the environment - relaxes the metabolic burden on any one microbe in the community (Freilich et al., 2011; Figure 1C). Given the complexity of microbial communities, each form of nutritional interaction may have ripple effects on other community members.

\section{COFACTOR CROSS-FEEDING}

In this review, we focus on cross-feeding of cofactors as a model for nutritional interactions between microbes. While cofactor cross-feeding is not necessarily mutualistic, the availability of exogenously produced cofactors can have a profound impact on a microbe's mode of growth and metabolite production. (Graber and Breznak, 2005; Pedersen etal., 2012). Examining cofactor cross-feeding can inform our understanding of the scope and relevance of metabolic interdependences in microbial communities.

\section{CROSS-FEEDING OF HEME AND QUINONES ALLOWS LACTIC ACID BACTERIA TO RESPIRE}

A striking example of the impact of cofactor cross-feeding on metabolism comes from studies of the lactic acid bacteria, (LAB). Though LAB were long considered to be exclusively dependent on fermentative growth, physiological changes in cultures grown with heme led to the hypothesis that some LAB are capable of aerobic respiration (Bryan-Jones and Whittenbury, 1969). Examination of sequenced environmental and host-associated LAB predicts that the majority have the genetic capacity for respiratory growth if heme, and in some cases, quinones, are supplied. The ability to respire in the presence of these cofactors are supplied has been experimentally verified in several LAB. Respiratory metabolism in LAB has been associated with increased growth rate, long-term survival, and a variety of metabolic changes that may impact other organisms in the environment (Pedersen etal., 2012). For example, in Lactococcus lactis (strains of which are frequently isolated from plants) and a number of other $\mathrm{LAB}$, switching from fermentation to respiratory metabolism leads to a decrease in the amount of lactic acid produced and a large increase in the production of acetoin, a volatile compound known to stimulate growth and induce the stringent response in plants (Duwat etal., 2001; Ryu et al., 2003; Rudrappa et al., 2010; Siezen et al., 2010; Jaaskelainen et al., 2013). In the opportunistic pathogen Streptococcus agalactiae, the ability to perform respiratory metabolism in the presence of heme and quinones enhances virulence and persistence (Rezaiki et al., 2008). Examples such as these, in which respiratory metabolism is determined 

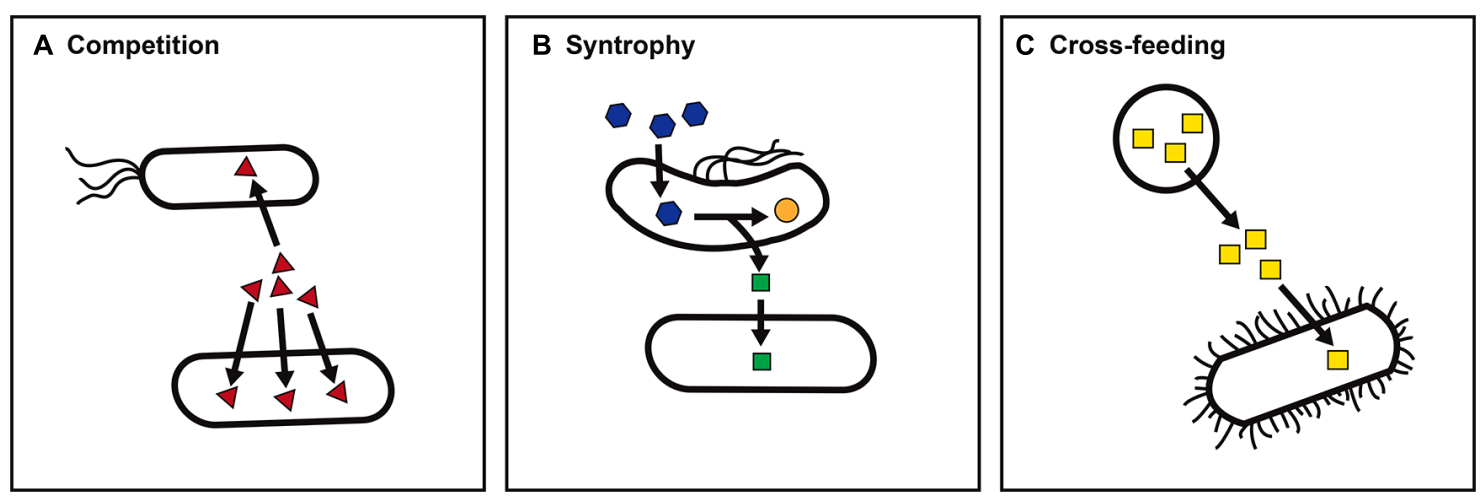

FIGURE 1 | Nutritional interactions between microbes. Nutritional interactions may shape a microbe's metabolic capacity within a microbial community. Three general categories of nutritional interactions are illustrated. (A) Nutrient competition. The ability of a microbe to compete for limiting nutrients such as iron (red triangles) may determine survival and persistence within a particular niche. (B) Syntrophic metabolism. The consumption of an intermediate or end product such as hydrogen (green squares) by a partner organism allows an otherwise energetically unfavorable reaction, for example, propionate (blue hexagons) to acetate (orange circles) to support growth. (C) Nutrient cross-feeding. The presence of a microbe that produces an essential nutrient such as folate (yellow squares) enables auxotrophs to survive. by the availability of exogenous cofactors, challenge our understanding of the metabolic capacity of microbes in their natural environments.

\section{COFACTOR CROSS-FEEDING IN THE TERMITE GUT: Treponema primitia AS BOTH A DONOR AND RECIPIENT OF COFACTORS}

Investigations of the termite gut-associated bacterium Treponema primitia illustrate the complex web of interactions that can exist within a host's microbiota. T. primitia contributes to the host's nutrition by producing acetate, the major carbon and energy source for the termite, by consuming $\mathrm{H}_{2}$ and $\mathrm{CO}_{2}$, which are generated as waste products by cellulolytic protists. The process of homoacetogenesis requires folate, yet T. primitia is incapable of synthesizing this cofactor (Graber and Breznak, 2004). Given that insects are not known to synthesize folate, and the folate content of the termite's diet is very low, T. primitia's folate requirements must be fulfilled by other members of the termite gut microbiota. L. lactis and Serratia grimesii isolated from the termite gut were identified as candidates for this role, as both bacteria secrete 5formyltetrahydrofolate, the dominant folate compound found in the gut, at levels capable of supporting T. primitia growth in vitro (Graber and Breznak, 2005).

T. primitia is likely to function as a donor of cofactors as well as a recipient. Cofactors produced by $T$. primitia enhance the growth of another important member of the termite gut microbiota, Treponema azotonutricium, which supplements the host's nitrogen-poor wood diet through nitrogen fixation (Graber et al., 2004). In co-culture, T. primitia and T. azotonutricium achieve higher growth rates and yields than either species grown in isolation. RNA-seq data suggest that in addition to interspecies hydrogen transfer between the two organisms, growth enhancement seen in T. azotonutricium may also be influenced by the production of the cofactors biotin, pyridoxal phosphate and co-enzyme A by T. primitia (Rosenthal et al., 2011). These findings undoubtedly represent only a small fraction of the complex interactions between members of a very diverse microbiota and provide clues about how microbes that are essential to a host's nutritional status support and are supported by other members of the microbial community.

\section{MODELS OF COFACTOR CROSS-FEEDING: CORRINOID SHARING IN MUTUALISTIC PAIRS AND MICROBIAL COMMUNITIES}

Of the many documented examples of nutrient cross-feeding, one group of cofactors, the corrinoids, has been particularly informative for understanding cross-feeding mechanisms. Corrinoid-dependent reactions function in diverse metabolic processes across all three domains of life, yet corrinoids are produced solely by a subset of prokaryotes. While the majority of bacteria $(75 \%)$ are predicted to encode corrinoid-dependent enzymes, at least half of these lack the ability to produce corrinoids de novo (Rodionov et al., 2003; Zhang et al., 2009). As such, corrinoid cross-feeding is prevalent and may reflect the advantage of acquiring these complex cofactors from the environment rather than by de novo biosynthesis which requires approximately 30 enzymatic steps (Roth etal., 1996; Warren etal., 2002; Zhang et al., 2009). The availability of corrinoids and corrinoid precursors can have profound effects on a microbe's metabolism and its ability to occupy a specific niche (Matthews, 2009). For instance, the ability to utilize ethanolamine as a sole carbon and nitrogen source relies on the corrinoid-dependent enzyme ethanolamine ammonia lyase, which converts ethanolamine into ammonia and acetaldehyde (Garsin, 2010). In enterohemorrhagic Escherichia coli, ethanolamine utilization - enabled by corrinoid cross-feeding - provides a competitive advantage for colonization and persistence in the bovine intestine, a main reservoir for this pathogen (Bertin et al., 2011; Kendall et al., 2012). Ethanolamine utilization is also important in other human pathogens that rely on exogenously produced corrinoids or corrinoid precursors including Listeria monocytogenes and Enterococcus faecalis (Joseph et al., 2006; Del Papa and Perego, 2008; Garsin, 
2010). The mechanism by which corrinoids are released from corrinoid-producing microbes into the environment is unclear. As yet, no active means of corrinoid export has been identified.

Sixteen distinct corrinoids with structural differences in the lower ligand have been identified and the array of corrinoids that can serve as cofactors differs from organism to organism (Barker et al., 1960; Renz, 1999; Hibbing et al., 2010; Yi et al., 2012). Given that microbial communities have been found to contain multiple corrinoids (Allen and Stabler, 2008; Girard etal., 2009; Men etal., 2014), how do corrinoid-dependent microbes acquire the specific corrinoids that function in their metabolism? As described below, some organisms form mutualisms with corrinoid-producing partners. Others may selectively import corrinoids from the environment or modify imported corrinoids or corrinoid precursors intracellularly (Figure 2).

\section{EXAMPLES OF SPECIFIC MUTUALISTIC INTERACTIONS} INVOLVING CORRINOIDS

Phytoplankton are of great ecological importance in their roles in the global carbon cycle, as primary producers in the marine food web, and occasionally as producers of toxins in harmful algal blooms (HABs). Half of eukaryotic algal species are predicted to require exogenously produced corrinoids for growth (Croft et al., 2005; Helliwell et al., 2011). Algal corrinoid requirements can be fulfilled via symbiotic relationships with corrinoid-producing bacteria that colonize the algal surface (Croft et al., 2005; Grant et al., 2014). The availability of corrinoids may play an important role in the occurrence of HABs, as corrinoid auxotrophy is especially prevalent among HAB species (Tang et al., 2010).

Symbiotic relationships can also be fueled by cross-feeding of products of corrinoid-dependent enzymes. Genomic studies of the cicada endosymbiotic bacteria, Hodgkinia cicadicola and

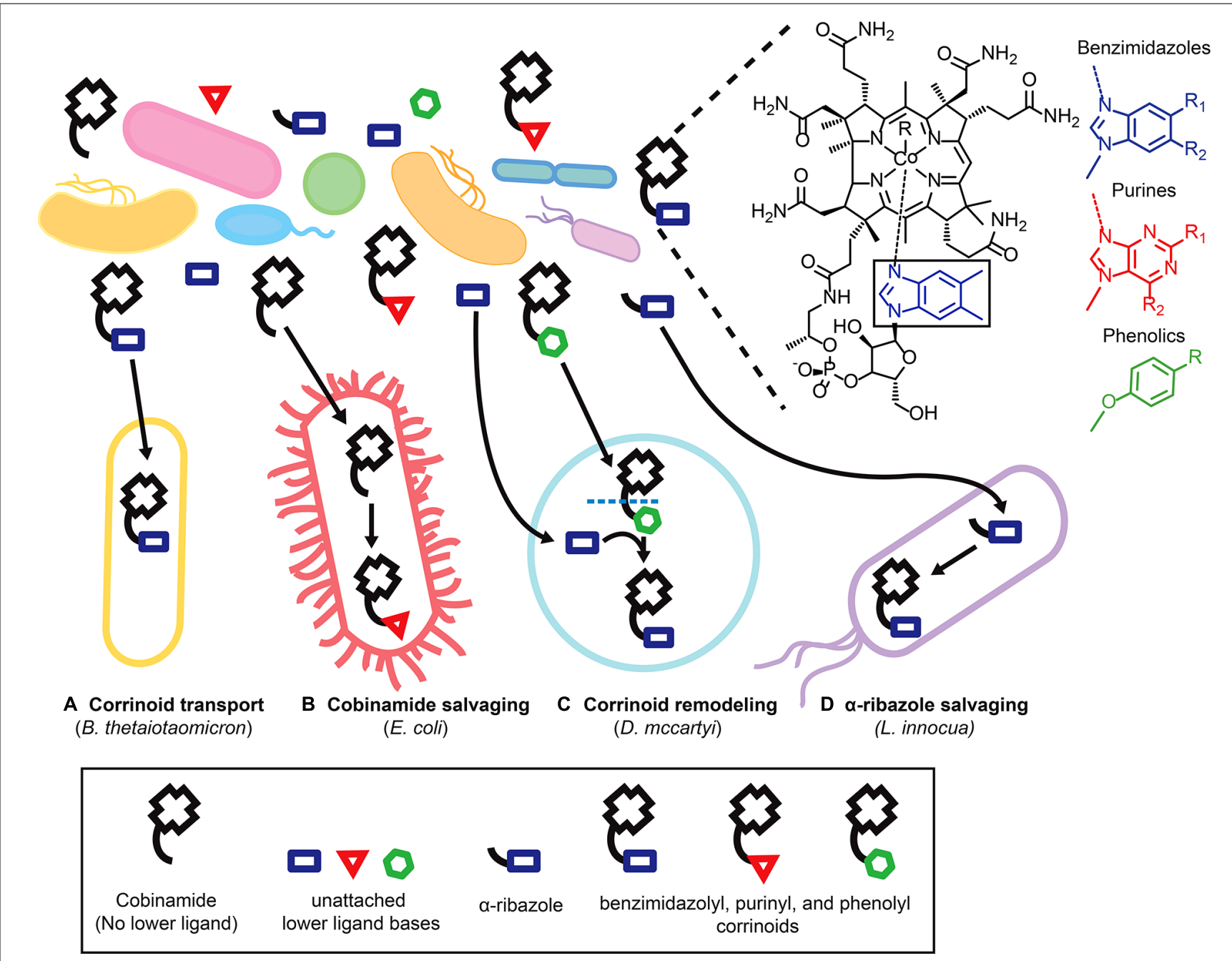

FIGURE 2 | Microbial strategies for fulfilling corrinoid requirements. Microbes employ various strategies to obtain specific corrinoids from environments that contain a variety of corrinoids and corrinoid precursors. The structure of cobalamin, a corrinoid with the lower ligand 5,6dimethylbenzimidazole (boxed) is shown, as are the structures of the three classes of lower ligand bases of other corrinoids. Specific strategies for obtaining corrinoids are illustrated with representative bacteria listed in parentheses. (A) Corrinoid transport in Bacteroides thetaiotaomicron. (B) Cobinamide salvaging in Escherichia coli. (C) Corrinoid remodeling in Dehalococcoides mccartyi. (D) $\alpha$-ribazole salvaging in Listeria innocua. 
Sulcia muelleri, indicate that the ability to produce a corrinoid is vital for maintaining this tripartite symbiosis (McCutcheon et al., 2009). H. cicadicola and S. muelleri together produce essential amino acids that are cross-fed between the co-symbionts and provided to the host. The burden of methionine production rests on $H$. cicadicola, which devotes $7 \%$ of its $144 \mathrm{~kb}$ genome to corrinoid biosynthesis because the methionine synthase it encodes requires a corrinoid cofactor. Interestingly, the corrinoid biosynthesis pathway in $H$. cicadicola is incomplete, and lacks genes involved in the production of precursors common to the biosynthesis of corrinoids as well as other tetrapyrroles such as heme (McCutcheon et al., 2009). We speculate that $H$. cicadicola may acquire tetrapyrrole precursors from the host, completing a loop between organisms which allows maintenance of the symbiotic relationship.

\section{STRATEGIES USED BY BACTERIA THAT SCAVENGE CORRINOIDS AND THEIR PRECURSORS FROM ENVIRONMENTAL SOURCES}

The ability of bacteria to selectively transport specific corrinoids has been largely unexplored, yet may play an important role in ensuring that bacteria acquire the specific corrinoids that function in their metabolism. The corrinoid transporter - BtuBFCD in gram negative bacteria, and BtuFCD in gram positive bacteria - allows bacteria to import corrinoids from the environment (Figure 2A) and is present in an estimated $76 \%$ of bacterial genomes (Zhang etal., 2009). A recent study of the human gut microbiome found that many bacteria encode multiple copies of the BtuBFCD corrinoid transporter, and that the three homologs of the outer membrane transporter BtuB encoded by the model gut bacterium Bacteroides thetaiotaomicron, though apparently redundant, have distinct preferences for different corrinoids (Degnan et al., 2014). The presence of multiple corrinoid transport systems with different affinities for specific corrinoids may allow bacteria to fine-tune their responses to the array of corrinoids present in the environment.

In contrast to obtaining specific corrinoids through selective transport, the ability to remodel corrinoids (that is, to remove the lower ligand of an imported corrinoid and attach a preferred lower ligand in its place) can enable microbes to make use of many different corrinoids present in a community (Gray and Escalante-Semerena, 2009). For instance, our recent work showed that Dehalococcoides mccartyi, which has an obligate requirement for exogenously produced corrinoids as cofactors in the reductive dehalogenation of the common groundwater pollutants tetrachloroethene (PCE) and trichloroethene (TCE), is restricted to the use of just three benzimidazolyl corrinoids (Maymo-Gatell et al., 1997; He et al., 2007; Yi et al., 2012). However, if an appropriate benzimidazole lower ligand base is supplied, D. mccartyi can fulfill its corrinoid requirements by remodeling other corrinoids (Figure 2C; Yi et al., 2012). Since benzimidazolyl corrinoids represent only a fraction of the corrinoids present in a community containing $D$. mccartyi, the ability to remodel corrinoids may be essential to its survival in the environment (Men etal., 2014). Corrinoid remodeling is also performed by some bacteria capable of producing a corrinoid de novo (Gray and Escalante-Semerena, 2009), and has been observed in the human gut, where examination of fecal corrinoid profiles before and after ingestion of cobalamin suggests that at least some members of the gut microbiota are engaged in active modification of exogenous corrinoids (Allen and Stabler, 2008).

In addition to importing complete corrinoids (i.e., those containing a lower ligand), some microbes are capable of importing corrinoid precursors from the environment and carrying out the remaining biosynthetic steps to produce a complete corrinoid. For example, though E. coli is incapable of de novo corrinoid biosynthesis, it possesses the ability to take up cobinamide (a corrinoid precursor that lacks a lower ligand) and convert it to a complete corrinoid through a process known as cobinamide salvaging (Figure 2B; Di Girolamo and Bradbeer, 1971; Raux et al., 1996; Escalante-Semerena, 2007). In contrast, Listeria spp. encode a nearly complete corrinoid biosynthesis pathway, but apparently lack the genes necessary for lower ligand activation, a step that must be completed before the lower ligand can be attached. Listeria innocua was shown instead to rely on the cblT encoded transporter to take up activated lower ligands such as $\alpha$ ribazole from the environment, which it then phosphorylates and attaches to produce a complete corrinoid (Gray and EscalanteSemerena, 2010; Figure 2D). CblT homologs have been identified in a variety of human pathogens including L. monocytogenes and Clostridium botulinum (Gray and Escalante-Semerena, 2010).

Microbes that produce corrinoids de novo can also be affected by the presence of corrinoid precursors. Guided biosynthesis, the process of controlling which corrinoid a microbe produces by providing an excess of a particular lower ligand base, can be a useful tool for determining the specific corrinoids required in different metabolic pathways (Stupperich et al., 1987; Keller et al., 2013). For example, in Sporomusa ovata, which produces phenolyl corrinoids, the addition of benzimidazoles and their subsequent incorporation into benzimidazolyl corrinoids inhibits growth to different degrees on substrates that require a corrinoid-dependent methyltransferase for utilization (Stupperich and Konle, 1993; Mok and Taga, 2013). We have detected free lower ligand bases in a variety of environmental samples, which raises the question of whether the production of a free lower ligand base by one organism is capable of affecting the corrinoid production of another; that is, whether guided biosynthesis occurs in nature. Evidence from our recent study of the corrinoid and lower ligand profiles in a TCE-dechlorinating community containing $D$. mccartyi suggests that this indeed may be the case (Men et al., 2014).

\section{CORRINOIDS AS LYNCHPINS OF MICROBIAL COMMUNITY DYNAMICS?}

Given that the majority of bacteria depend on corrinoids for their metabolism, and that only a fraction of available corrinoids may be suitable for use by a particular organism, could it be possible to manipulate microbial communities by targeting corrinoid-dependent metabolism? Recent work has shown that manipulation of the composition of a TCE-dechlorinating community can result in a shift in corrinoid composition, and conversely, that the addition of a corrinoid causes a dramatic shift in marine algal community 
composition (Koch et al., 2011; Men et al., 2014). With growing interest in developing methods for targeted manipulation of microbial communities to benefit human health and the environment, the utility of altering the composition and/or metabolism occurring in microbial communities via corrinoid supplementation or guided biosynthesis deserves further investigation.

\section{CONCLUSION}

From obligate requirements for exogenously supplied cofactors in T. primitia and D. mccartyi to unlocking cryptic modes of growth in $\mathrm{LAB}$, examples of cofactor cross-feeding provide a glimpse into the impact of nutritional interactions on individual species as well as entire communities. Cofactor cross-feeding undoubtedly deserves further examination; one need look no further than the vitamin amendments required for microbes growing in pure culture to obtain examples of nutrients that must be supplied by other organisms in their natural environment. Advances in imaging mass spectrometry and multiple "omics" approaches, in combination with pure culture studies of individual microbes and defined consortia, bring us closer to understanding the molecular details of metabolic interactions at new levels (Nakanishi et al., 2011; Phelan et al., 2012).

\section{ACKNOWLEDGMENT}

This work was supported by National Science Foundation grant MCB1122046 to Michiko E. Taga.

\section{REFERENCES}

Allen, R. H., and Stabler, S. P. (2008). Identification and quantitation of cobalamin and cobalamin analogs in human feces. Am. J. Clin. Nutr. 87, 1324-1335.

Barker, H. A., Smyth, R. D., Weissbach, H., Toohey, J. I., Ladd, J. N., and Volcani, B. E. (1960). Isolation and properties of crystalline cobamide coenzymes containing benzimidazole or 5, 6-dimethylbenzimidazole. J. Biol. Chem. 235, 480-488.

Bertin, Y., Girardeau, J. P., Chaucheyras-Durand, F., Lyan, B., Pujos-Guillot, E., Harel, J., et al. (2011). Enterohaemorrhagic Escherichia coli gains a competitive advantage by using ethanolamine as a nitrogen source in the bovine intestinal content. Environ. Microbiol. 13, 365-377. doi: 10.1111/j.1462-2920.2010. 02334.x

Bryan-Jones, D. G., and Whittenbury, R. (1969). Haematin-dependent oxidative phosphorylation in Streptococcus faecalis. J. Gen. Microbiol. 58, 247-260. doi: 10.1099/00221287-58-2-247

Croft, M. T., Lawrence, A. D., Raux-Deery, E., Warren, M. J., and Smith, A. G. (2005). Algae acquire vitamin B12 through a symbiotic relationship with bacteria. Nature 438, 90-93. doi: 10.1038/nature04056

Degnan, P. H., Barry, N. A., Mok, K. C., Taga, M. E., and Goodman, A. L. (2014). Human gut microbes use multiple transporters to distinguish vitamin B12 analogs and compete in the gut. Cell Host Microbe 15, 47-57. doi: 10.1016/j.chom.2013.12.007

Del Papa, M. F., and Perego, M. (2008). Ethanolamine activates a sensor histidine kinase regulating its utilization in Enterococcus faecalis. J. Bacteriol. 190, 7147 7156. doi: 10.1128/JB.00952-08

Di Girolamo, P. M., and Bradbeer, C. (1971). Transport of vitamin B12 in Escherichia coli. J. Bacteriol. 106, 745-750.

Duwat, P., Sourice, S., Cesselin, B., Lamberet, G., Vido, K., Gaudu, P., et al. (2001). Respiration capacity of the fermenting bacterium Lactococcus lactis and its positive effects on growth and survival. J. Bacteriol. 183, 4509-4516. doi: 10.1128/JB.183.15.4509-4516.2001

Escalante-Semerena, J. C. (2007). Conversion of cobinamide into adenosylcobamide in bacteria and archaea. J. Bacteriol. 189, 4555-4560. doi: 10.1128/JB.00503-07

Faust, K., and Raes, J. (2012). Microbial interactions: from networks to models. Nat. Rev. Microbiol. 10, 538-550. doi: 10.1038/nrmicro2832
Freilich, S., Zarecki, R., Eilam, O., Segal, E. S., Henry, C. S., Kupiec, M., et al. (2011). Competitive and cooperative metabolic interactions in bacterial communities. Nat. Commun. 2:589. doi: 10.1038/ncomms1597

Garsin, D. A. (2010). Ethanolamine utilization in bacterial pathogens: roles and regulation. Nat. Rev. Microbiol. 8, 290-295. doi: 10.1038/nrmicro2334

Girard, C. L., Santschi, D. E., Stabler, S. P., and Allen, R. H. (2009). Apparent ruminal synthesis and intestinal disappearance of vitamin B12 and its analogs in dairy cows. J. Dairy Sci. 92, 4524-4529. doi: 10.3168/jds.2009-2049

Graber, J. R., and Breznak, J. A. (2004). Physiology and nutrition of Treponema primitia, an $\mathrm{H} 2 / \mathrm{CO} 2-$ acetogenic spirochete from termite hindguts. Appl. Environ. Microbiol. 70, 1307-1314. doi: 10.1128/AEM.70.3.1307-1314.2004

Graber, J. R., and Breznak, J. A. (2005). Folate cross-feeding supports symbiotic homoacetogenic spirochetes. Appl. Environ. Microbiol. 71, 1883-1889. doi: 10.1128/AEM.71.4.1883-1889.2005

Graber, J. R., Leadbetter, J. R., and Breznak, J. A. (2004). Description of Treponema azotonutricium sp. nov. and Treponema primitia sp. nov., the first spirochetes isolated from termite guts. Appl. Environ. Microbiol. 70, 1315-1320. doi: 10.1128/aem.70.3.1315-1320.2004

Grant, M. A., Kazamia, E., Cicuta, P., and Smith, A. G. (2014). Direct exchange of vitamin B12 is demonstrated by modeling the growth dynamics of algal-bacterial cocultures. ISME J. 8, 1418-1427. doi: 10.1038/ismej.2014.9

Gray, M. J., and Escalante-Semerena, J. C. (2009). The cobinamide amidohydrolase (cobyric acid-forming) CbiZ enzyme: a critical activity of the cobamide remodeling system of Rhodobacter sphaeroides. Mol. Microbiol. 74, 1198-1210. doi: 10.1111/j.1365-2958.2009.06928.x

Gray, M. J., and Escalante-Semerena, J. C. (2010). A new pathway for the synthesis of alpha-ribazole-phosphate in Listeria innocua. Mol. Microbiol. 77, 1429-1438. doi: 10.1111/j.1365-2958.2010.07294.x

He, J., Holmes, V. F., Lee, P. K., and Alvarez-Cohen, L. (2007). Influence of vitamin B12 and cocultures on the growth of Dehalococcoides isolates in defined medium. Appl. Environ. Microbiol. 73, 2847-2853. doi: 10.1128/AEM.02574-06

Helliwell, K. E., Wheeler, G. L., Leptos, K. C., Goldstein, R. E., and Smith, A. G. (2011). Insights into the evolution of vitamin B12 auxotrophy from sequenced algal genomes. Mol. Biol. Evol. 28, 2921-2933. doi: 10.1093/molbev/msr 124

Hibbing, M. E., Fuqua, C., Parsek, M. R., and Peterson, S. B. (2010). Bacterial competition: surviving and thriving in the microbial jungle. Nat. Rev. Microbiol. 8, 15-25. doi: 10.1038/nrmicro2259

Jaaskelainen, E., Johansson, P., Kostiainen, O., Nieminen, T., Schmidt, G., Somervuo, P., et al. (2013). Significance of heme-based respiration in meat spoilage caused by Leuconostoc gasicomitatum. Appl. Environ. Microbiol. 79, 1078-1085. doi: 10.1128/AEM.02943-12

Johnson, D. R., Goldschmidt, F., Lilja, E. E., and Ackermann, M. (2012). Metabolic specialization and the assembly of microbial communities. ISME J. 6, 1985-1991. doi: 10.1038/ismej.2012.46

Joseph, B., Przybilla, K., Stuhler, C., Schauer, K., Slaghuis, J., Fuchs, T. M., et al. (2006). Identification of Listeria monocytogenes genes contributing to intracellular replication by expression profiling and mutant screening. J. Bacteriol. 188, 556568. doi: 10.1128/JB.188.2.556-568.2006

Keller, S., Ruetz, M., Kunze, C., Krautler, B., Diekert, G., and Schubert, T. (2013). Exogenous 5,6-dimethylbenzimidazole caused production of a nonfunctional tetrachloroethene reductive dehalogenase in Sulfurospirillum multivorans. Environ. Microbiol. doi: 10.1111/1462-2920.12268 [Epub ahead of print].

Kendall, M. M., Gruber, C. C., Parker, C. T., and Sperandio, V. (2012). Ethanolamine controls expression of genes encoding components involved in interkingdom signaling and virulence in enterohemorrhagic Escherichia coli O157:H7. MBio 3. doi: $10.1128 / \mathrm{mBio} .00050-12$

Koch, F., Alejandra Marcoval, M., Panzeca, C., Bruland, K. W., Sañudo-Wilhelmy, S. A., and Gobler, C. J. (2011). The effect of vitamin B12 on phytoplankton growth and community structure in the Gulf of Alaska. Limnol. Oceanogr. 56, 1023-1034. doi: 10.4319/lo.2011.56.3.1023

Leadbetter, J. R. (2003). Cultivation of recalcitrant microbes: cells are alive, well and revealing their secrets in the 21st century laboratory. Curr. Opin. Microbiol. 6, 274-281. doi: 10.1016/s1369-5274(03)00041-49

Matthews, R. G. (2009). Cobalamin- and corrinoid-dependent enzymes. Met. Ions Life Sci. 6, 53-114. doi: 10.1039/BK9781847559159-00053

Maymo-Gatell, X., Chien, Y., Gossett, J. M., and Zinder, S. H. (1997). Isolation of a bacterium that reductively dechlorinates tetrachloroethene 
to ethene. Science 276, 1568-1571. doi: 10.1126/science.276.5318. 1568

McCutcheon, J. P., Mcdonald, B. R., and Moran, N. A. (2009). Convergent evolution of metabolic roles in bacterial co-symbionts of insects. Proc. Natl. Acad. Sci. U.S.A. 106, 15394-15399. doi: 10.1073/pnas.0906424106

Men, Y., Seth, E. C., Yi, S., Crofts, T. S., Allen, R. H., Taga, M. E., et al. (2014). Identification of specific corrinoids reveals corrinoid modification in dechlorinating microbial communities. Environ. Microbiol. doi: 10.1111/1462-2920.12500 [Epub ahead of print].

Mok, K. C., and Taga, M. E. (2013). Growth inhibition of Sporomusa ovata by incorporation of benzimidazole bases into cobamides. J. Bacteriol. 195, 19021911. doi: 10.1128/JB.01282-12

Moller, S., Sternberg, C., Andersen, J. B., Christensen, B. B., Ramos, J. L., Givskov, M., et al. (1998). In situ gene expression in mixed-culture biofilms: evidence of metabolic interactions between community members. Appl. Environ. Microbiol. 64, 721-732.

Morris, B. E., Henneberger, R., Huber, H., and Moissl-Eichinger, C. (2013). Microbial syntrophy: interaction for the common good. FEMS Microbiol. Rev. 37, 384-406. doi: 10.1111/1574-6976.12019

Nakanishi, Y., Fukuda, S., Chikayama, E., Kimura, Y., Ohno, H., and Kikuchi, J. (2011). Dynamic omics approach identifies nutrition-mediated microbial interactions. J. Proteome Res. 10, 824-836. doi: 10.1021/pr100989c

Pedersen, M. B., Gaudu, P., Lechardeur, D., Petit, M. A., and Gruss, A. (2012). Aerobic respiration metabolism in lactic acid bacteria and uses in biotechnology. Annu. Rev. Food Sci. Technol. 3, 37-58. doi: 10.1146/annurev-food-022811-101255

Phelan, V. V., Liu, W. T., Pogliano, K., and Dorrestein, P. C. (2012). Microbial metabolic exchange-the chemotype-to-phenotype link. Nat. Chem. Biol. 8, 2635. doi: 10.1038/nchembio.739

Raux, E., Lanois, A., Levillayer, F., Warren, M. J., Brody, E., Rambach, A., et al. (1996). Salmonella typhimurium cobalamin (vitamin B12) biosynthetic genes: functional studies in S. typhimurium and Escherichia coli. J. Bacteriol. 178, 753-767.

Renz, P. (1999). "Biosynthesis of the 5,6-dimethylbenzimidazole moiety of cobalamin and of the other bases found in natural corrinoids," in Chemistry and Biochemistry of B12, ed. R Banerjee (New York, NY: John Wiley \& Sons, Inc.), 557-575.

Rezaiki, L., Lamberet, G., Derre, A., Gruss, A., and Gaudu, P. (2008). Lactococcus lactis produces short-chain quinones that cross-feed Group B Streptococcus to activate respiration growth. Mol. Microbiol. 67, 947-957. doi: 10.1111/j.13652958.2007.06083.x

Rodionov, D. A., Vitreschak, A. G., Mironov, A. A., and Gelfand, M. S. (2003). Comparative genomics of the vitamin B12 metabolism and regulation in prokaryotes. J. Biol. Chem. 278, 41148-41159. doi: 10.1074/jbc.M305837200 [Epub ahead of print].

Rosenthal, A. Z., Matson, E. G., Eldar, A., and Leadbetter, J. R. (2011). RNA-seq reveals cooperative metabolic interactions between two termite-gut spirochete species in co-culture. ISME J. 5, 1133-1142. doi: 10.1038/ismej.2011.3

Roth, J. R., Lawrence, J. G., and Bobik, T. A. (1996). Cobalamin (coenzyme B12): synthesis and biological significance. Annu. Rev. Microbiol. 50, 137-181. doi: 10.1146/annurev.micro.50.1.137

Rudrappa, T., Biedrzycki, M. L., Kunjeti, S. G., Donofrio, N. M., Czymmek, K. J., Pare, P. W., et al. (2010). The rhizobacterial elicitor acetoin induces systemic resistance in Arabidopsis thaliana. Commun. Integr. Biol. 3, 130-138. doi: $10.4161 / \mathrm{cib} .3 .2 .10584$
Ryu, C. M., Farag, M. A., Hu, C. H., Reddy, M. S., Wei, H. X., Pare, P. W., etal. (2003). Bacterial volatiles promote growth in Arabidopsis. Proc. Natl. Acad. Sci. U.S.A. 100, 4927-4932. doi: 10.1073/pnas.0730 845100

Sieber, J. R., Mcinerney, M. J., and Gunsalus, R. P. (2012). Genomic insights into syntrophy: the paradigm for anaerobic metabolic cooperation. Annu. Rev. Microbiol. 66, 429-452. doi: 10.1146/annurev-micro-090110102844

Siezen, R. J., Bayjanov, J., Renckens, B., Wels, M., Van Hijum, S. A., Molenaar, D., et al. (2010). Complete genome sequence of Lactococcus lactis subsp. lactis KF147, a plant-associated lactic acid bacterium. J. Bacteriol. 192, 2649-2650. doi: 10.1128/JB.00276-210

Stupperich, E., and Konle, R. (1993). Corrinoid-dependent methyl transfer reactions are involved in methanol and 3,4-dimethoxybenzoate metabolism by Sporomusa ovata. Appl. Environ. Microbiol. 59, 3110-3116.

Stupperich, E., Steiner, I., and Eisinger, H. J. (1987). Substitution of Co alpha(5-hydroxybenzimidazolyl)cobamide (factor III) by vitamin B12 in Methanobacterium thermoautotrophicum. J. Bacteriol. 169, 3076-3081.

Tang, Y. Z., Koch, F., and Gobler, C. J. (2010). Most harmful algal bloom species are vitamin B1 and B12 auxotrophs. Proc. Natl. Acad. Sci. U.S.A. 107, 20756-20761. doi: 10.1073/pnas.1009566107

Traxler, M. F., Watrous, J. D., Alexandrov, T., Dorrestein, P. C., and Kolter, R. (2013). Interspecies interactions stimulate diversification of the Streptomyces coelicolor secreted metabolome. MBio 4. doi: 10.1128/mBio.00459-13

Tyson, G. W., and Banfield, J. F. (2005). Cultivating the uncultivated: a community genomics perspective. Trends Microbiol. 13, 411-415. doi: 10.1016/j.tim.2005.07.003

Warren, M. J., Raux, E., Schubert, H. L., and Escalante-Semerena, J. C. (2002). The biosynthesis of adenosylcobalamin (vitamin B12). Nat. Prod. Rep. 19, 390-412. doi: 10.1039/b108967f

Yi, S., Seth, E. C., Men, Y. J., Stabler, S. P., Allen, R. H., Alvarez-Cohen, L., et al. (2012). Versatility in corrinoid salvaging and remodeling pathways supports corrinoiddependent metabolism in Dehalococcoides mccartyi. Appl. Environ. Microbiol. 78, 7745-7752. doi: 10.1128/AEM.02150-12

Zhang, Y., Rodionov, D. A., Gelfand, M. S., and Gladyshev, V. N. (2009). Comparative genomic analyzes of nickel, cobalt and vitamin B12 utilization. BMC Genomics 10:78. doi: 10.1186/1471-2164-10-78

Conflict of Interest Statement: The authors declare that the research was conducted in the absence of any commercial or financial relationships that could be construed as a potential conflict of interest.

Received: 30 May 2014; accepted: 23 June 2014; published online: 08 July 2014. Citation: Seth EC and Taga ME (2014) Nutrient cross-feeding in the microbial world. Front. Microbiol. 5:350. doi: 10.3389/fmicb.2014.00350

This article was submitted to Terrestrial Microbiology, a section of the journal Frontiers in Microbiology.

Copyright () 2014 Seth and Taga. This is an open-access article distributed under the terms of the Creative Commons Attribution License (CC BY). The use, distribution or reproduction in other forums is permitted, provided the original author (s) or licensor are credited and that the original publication in this journal is cited, in accordance with accepted academic practice. No use, distribution or reproduction is permitted which does not comply with these terms. 